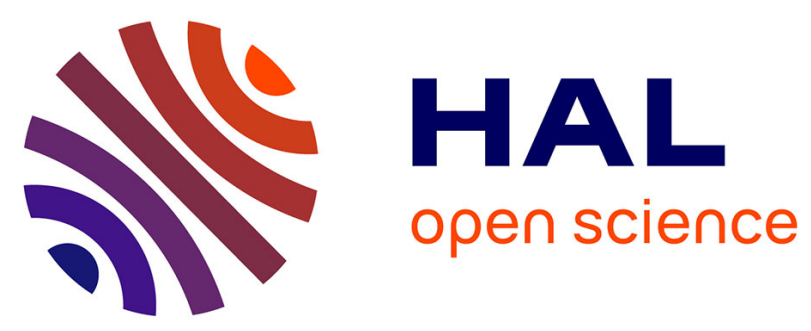

\title{
Resonance fluorescence of a single semiconductor quantum dot: the impact of a fluctuating electrostatic environment
}

\author{
Antoine Reigue, Richard Hostein, Valia Voliotis
}

\section{- To cite this version:}

Antoine Reigue, Richard Hostein, Valia Voliotis. Resonance fluorescence of a single semiconductor quantum dot: the impact of a fluctuating electrostatic environment. Semiconductor Science and Technology, 2019, 34 (11), pp.113001. 10.1088/1361-6641/ab4362 . hal-02404993

\section{HAL Id: hal-02404993 \\ https://hal.sorbonne-universite.fr/hal-02404993}

Submitted on 11 Dec 2019

HAL is a multi-disciplinary open access archive for the deposit and dissemination of scientific research documents, whether they are published or not. The documents may come from teaching and research institutions in France or abroad, or from public or private research centers.
L'archive ouverte pluridisciplinaire HAL, est destinée au dépôt et à la diffusion de documents scientifiques de niveau recherche, publiés ou non, émanant des établissements d'enseignement et de recherche français ou étrangers, des laboratoires publics ou privés. 


\title{
Resonance fluorescence of a single semiconductor quantum dot : the impact of a fluctuating electrostatic environment
}

\author{
Antoine Reigue, ${ }^{1}$ Richard Hostein, ${ }^{2}$ and Valia Voliotis ${ }^{2}$, * \\ ${ }^{1}$ Institut Néel, Université Grenoble Alpes, CNRS UPR2940, 38042 Grenoble, France \\ ${ }^{2}$ Sorbonne Université, CNRS, Institut des NanoSciences de Paris, INSP, 75005-Paris, France
}

(Dated: August 30, 2019)

\begin{abstract}
Semiconductor quantum dots are very efficient sources of single and highly indistinguishable photons. These properties rely on the possibility to coherently control the system at the single spin level. At this ultimate level of control, the quantum dot becomes a very sensitive probe of its solid-state environment and any interaction turns into a dephasing process that alters its coherence properties. In this topical review, we give an overview of the issue of charge noise which remains one of the main dephasing mechanisms to overcome. This phenomenon which strongly depends on sample preparation, originates from a fluctuating electrostatic landscape around the quantum dots and renders a unified description quite awkward. We present the common characteristic features induced by charge noise that have been observed in the resonant fluorescence experiments of single quantum dots and discuss the different approaches that have been proposed in the literature to circumvent this problem.
\end{abstract}

\section{INTRODUCTION}

Semiconductor quantum dots are among the most popular and mature solid-state quantum systems for implementing stationary qubits for future applications in quantum information technologies 1 . Their ease integration into smartly designed nanophotonic devices ${ }^{2}$ has lead to important breakthroughs especially for highly efficient on-demand generation of single photons ${ }^{3}[6$ with a high degree of indistinguishability ${ }^{7-10}$.

Despite these achievements, there is still a lack of control of the quantum dot (QD) interaction with its solid environment which strongly influences photon emission and is detrimental to its coherence properties. An inherent problem related to the growth process of semiconductor QDs is the presence of residual doping 11 that might lead to trapping of carriers on localization sites created by different point defects in the samples ${ }^{12}$ inducing a fluctuating electrostatic environment in the vicinity of the dots.

The resulting electrostatic field leads to quantum confined Stark effect and its fluctuations in time give rise to spectral diffusion or spectral wandering of the emission lines. This phenomenon is observed in many systems where the optical properties are dominated by emission from three-dimensional confined states like in quantum $\operatorname{dots}_{15} 23$, colloidal nanocrystals 24,26 , molecules 2728 or NV centers in diamond 29 . The fluctuating electrostatic field impacts the emission properties by inducing intermittent emission or blinking 30 , inhomogeneous spectral broadening and as a consequence dephasing of the optically addressed quantum state.

Resonant excitation $\sqrt[3132]{ }$ has been proposed to suppress some of the occurring dephasing processes, in particular those related to energy relaxation of carriers after their above-band photocreation and time-jitter in the state preparation. Resonance fluorescence became not only a powerful tool to study the intrinsic properties of single quantum dots but also a very sensitive probe of the interactions between the quantum system and its environment.

Nevertheless, because of the local charge environment fluctuations, optical transitions can be quenched even when excited on resonance. This situation can for instance prevent resonant absorption to occur due to Coulomb blockade effects 33 . To circumvent this difficulty and recover the resonance fluorescence (RF), two main approaches have been proposed in the literature: the use of (i) a weak off-resonant additional excitation source, such as a He-Ne laser that allows "photo-neutralization" of the charge environment of the dot, and (ii) a chargecontrolled device with an electrical biased structure to stabilize the electrostatic potential outside the dot and control the number of charges in it.

While the first approach has led to an unquestionable improvement of the emission process efficiency, the underlying physical mechanisms are not completely understood and seem to be sample-dependent. The second approach has the advantage to control the dot energy level occupancy and stabilize the resonantly photocreated carriers while keeping the coherence properties. Despite these achievements, truly radiative-limited emission has not yet been obtained in most of the QDs systems and the residual dephasing mechanisms need to be controlled.

Here, most of the presented experiments and relative discussions hold for optically addressed neutral or charged electron-hole (e-h) pairs in single self-assembled InAs/GaAs QDs which have been the most studied systems in the literature the past twenty years. GaAs QDs obtained by infilling of nanoholes $34 / 35$ present also important charge fluctuations in the vicinity of the dots induced by the droplet growth method as recently reported $23 \mid 36$. Therefore, we believe that the main features described here, can be of general interest for other three-dimensional confined systems.

In this review, we will focus on relevant experiments 
showing that a strong quenching of the resonant emission occurs in a large variety of samples. In section II, we give some general definition of the concept of coherence time, how it can be affected by the QD's environment and how we can optically probe it by non-resonant or resonant laser excitation. In section III, we go into the physical origin of the existing fluctuating electrostatic environment of QDs and the consequences on the coherence properties of their quantum states. Finally in section IV, we give an overview of the different ways that have been proposed to skirt the problem of charge noise. We conclude by discussing the state-of the art performances achieved in terms of the QD RF line broadening.

\section{QUANTUM DOT: AN OPEN QUANTUM SYSTEM}

The tremendous progress in crystal growth techniques in the last decades has opened up the way to probe isolated nanostructures like single semiconductor QDs and investigate their individual intrinsic electronic properties.

The discrete energy spectrum of a single QD evidenced experimentally $\sqrt{38}$, pushed the community to consider in a first approach the QD as an "artificial atom" and describe it as a simple two-level system 39 (TLS). However, this ideal representation does not reflect the reality of a system like a QD which is embedded in a crystalline matrix and interacts with its solid environment. It has rather to be considered as an open quantum system 40 coupled to a reservoir of phonons 4 , of nuclear spins 4 and a fluctuating electrostatic environment caused by the random trapping/escape of charge carriers in the vicinity of the dot $18|33| 4345$.

An immediate consequence of these unwanted interactions is that the linewidth of the QD emission spectrum is not only limited by the radiative lifetime $\mathrm{T}_{1}$ of the electronic excitation as for atoms $\sqrt{39}$, but has an additional broadening term linked to the dephasing mechanisms. We can then relate the total broadening of the emission line $\Delta \mathrm{E}=\hbar / \mathrm{T}_{2}$ to the "coherence time" $\mathrm{T}_{2}$ which is given by $1 / \mathrm{T}_{2}=1 / 2 \mathrm{~T}_{1}+1 / \mathrm{T}_{2}^{*}$, with $\mathrm{T}_{2}^{*}$ the so-called "pure dephasing" time ${ }^{46}$ related to the loss of coherence of the quantum system, without energy relaxation. $\mathrm{T}_{2}^{*}$ quantifies in some sort the deviation from an ideal two-level system where the linewidth would be radiatively-limited. Hence, two strategies can be adopted in order to preserve the coherence properties, either by increasing $\mathrm{T}_{2}^{*}$, or reducing the radiative lifetime $\mathrm{T}_{1}$ of the system. It means that either the dephasing mechanisms have to be controlled and minimized, or one has to take advantage of cavity quantum electrodynamics (CQED) effects.

The problem of coherence in QDs is a long-standing issue addressed first for QDs ensembles 49 51 and progressively for individual QD\$16/43|52/53. More recently, with the development of quantum technologies using semiconductor QDs, it appeared that radiatively-limited lines are highly desirable especially for the generation of entangled photon pairs $54 \mid 55$ or indistinguishable single photons 56 . Therefore a lot of efforts have been spend by the community in order to observe single Lorentzian homogeneous lines with a coherence time as close as possible to the radiative limit, with $\mathrm{T}_{2}=2 \mathrm{~T}_{1}$. Typical values of radiative lifetimes for a neutral e-h pair in self-assembled InAs/GaAs QDs range between a few hundreds of ps to one ns depending on whether the dots are embedded in a cavity or not ${ }^{110}$. Thus, the maximum achievable coherence time is of the order of $2 \mathrm{~ns}$ for an optically excited e-h pair. By using spin states as qubits, the coherence time can reach microseconds range for a resident carrier in the $\mathrm{QD} \sqrt[57+59]{5}$

Whatever the optical excitation scheme, non-resonant or resonant with the optical transitions, the dephasing due to interaction with the acoustic phonon bath is unavoidable even at low temperature. It has been extensively studied the past years and its impact on the coherence properties of QDs is now quite well understood $\sqrt{60} 63$. It induces a typical asymmetric broadening on both sides of the zero-phonon line of the RF spectrum (the so-called "phonon side-bands") and reduces the degree of indistinguishability of the emitted single photons without postselection ${ }^{61}$ to $85 \%$. Charge noise affects both the neutral and charged excitons ${ }^{111}$ while spin noise affects predominantly the charged electron-hole (e-h) pair because of the

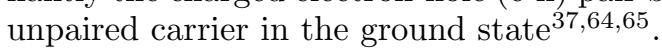

The coherence properties of an emitter are usually investigated through optical techniques with an excitation laser tuned off-resonance from the transition for commodity (see Fig 1(a)). However in this excitation scheme, the energy relaxation of the photocreated carriers from higher energy levels and the non-resonant capture in the dot $\frac{66}{6}$ reduces drastically the coherence time ${ }^{7}$, as shown in Fig 2. Early interferometric measurements showed an efficient line narrowing of the QD emission spectrum of 30 $\%$ as the excitation energy gets closer to the resonance ${ }^{43}$, leading to much longer coherence times, although not reaching the radiative limit.

Thus, resonant excitation and resonant detection of the luminescence of single QDs came to the fore as a way to keep the coherence properties of the emitter and consider it as a two-level system characterized by its radiatively-limited broadening. Resonant excitation was experimentally challenging to achieve because of the scattered excitation laser (see Fig 1 b-d). Specific sample geometries have been proposed to tackle this problem: QDs were embedded in planar microcavities 31 , one dimensional waveguides 32 , or micropillars $\$$, allowing for an orthogonal excitation/detection scheme and detecting resonant luminescence. In the usual backscattering geometry, it is then mandatory to use a dark-field technique with crossed linear polarizations for excitation and detection $\underline{68}$ to reject the resonant scattered laser. Figure 1 depicts some of the proposed excitation schemes to achieve resonant excitation and detection of single QD emission. 
(a)

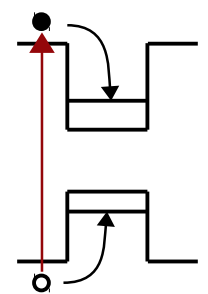

Non-resonant excitation (b)

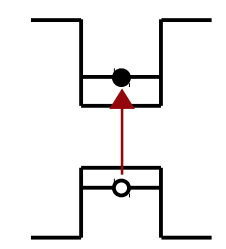

Resonant excitation (d)

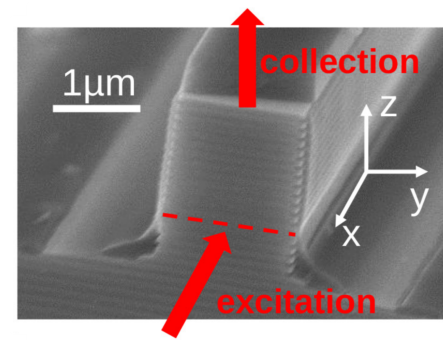

(c)

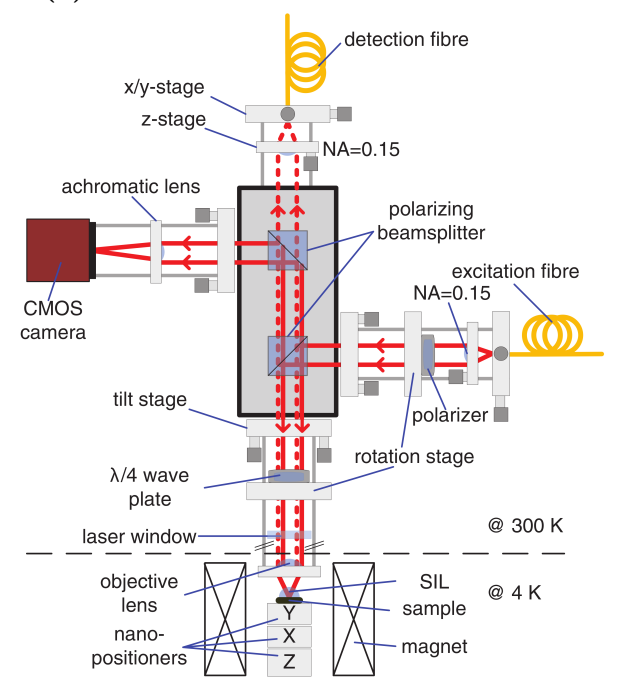

FIG. 1: Different optical excitation and detection schemes for spectroscopy of QDs. (a) In a non-resonant excitation, the laser wavelength is tuned to high energy continuum states, the photocreated e-h pairs relax non-radiatively by very efficient phonon processes into the QDs. (b) In a resonant excitation, the laser wavelength is tuned on resonance with a given optical transition. (c) Detection scheme for RF experiments where the laser suppression is implemented by means of orthogonal excitation/collection polarization states: the linear polarizer sets the laser polarization to s, matching the lower PBS; the quarter-wave plate controls the state of polarization; and the PBSs reject the s-polarized back-reflected laser light. Solid lines indicate s-polarization, dashed lines p-polarization. Figure reproduced from Ref [68]. (d) SEM image showing a $\mu \mathrm{m}$ ridge onedimensional waveguide used for RF experiments with orthogonal excitation and detection directions (courtesy K. Merghem, $\mathrm{C} 2 \mathrm{~N})$. The red dashed line represents the QDs plane embedded in Bragg mirrors.

Instead of probing the emission, other ways to investigate resonantly the QDs optical transitions are absorption experiments6971, or photocurrent measurements revealing nice features of TLS like in atoms. In photocurrent experiments, InGaAs/GaAs dots embedded in a ni-Schottky diode show many Rabi oscillations damped mainly by the coupling to acoustic phonons ${ }^{72 / 73}$. The effects of a fluctuating electrostatic environment are not mentioned in $\operatorname{Ref}\left[{ }^{72}\right]$ probably because charge noise is minimized in charge-controlled devices, as discussed in the next sections.

Even though resonant excitation has been successfully achieved, RF was not systematically observed. Only for a few percent of QDs on a sample, the luminescence was not quenched, and this observation was reported in many experiments performed with different samples with resonant pulsed or continuous-wave laser excitation 7/33/74 79 . Therefore, the naive picture of an ideal two-level system that can be resonantly excited, which in turn shields its intrinsic coherence properties from the coupling to the environment, breaks down. In what follows, we will show that RF quenching stems from the fluctuating electrostatic environment, that is also responsible for prominent dephasing due to Coulomb scattering.

\section{THE IMPACT OF A FLUCTUATING ELECTROSTATIC ENVIRONMENT}

The electrostatic landscape and the exact potential profile near each QD are quite complex to grasp, completely governed by the mechanisms of sample growth conditions. Therefore, it is a rather difficult task to draw a unified picture of the related dephasing phenomena, which also explains the diversity of results obtained for the coherence times even in resonant excitation conditions. Different scenarii have to be invoked, depending on sample growth conditions and experimental observations. Nevertheless, we will try in what follows to outline some general trends.

- Physical origin of charge noise in quantum dots. The unintentional background concentration during molecular beam epitaxy of GaAs-based materials is inevitable and for instance, carbon incorporation is known to be a residual source of acceptors $11 / 75$. This results in a background doping which constitutes an intrinsic reservoir of carriers if the impurities are ionized. These charge carriers or those photocreated during the optical excitation process can be trapped in deep levels in the barrier materials $53[80$ or in structural defects at the different heterointerfaces of the self-assembled InAs/GaAs QDs samples ${ }^{12}$ 14. Moreover if the QD layer is embedded in 


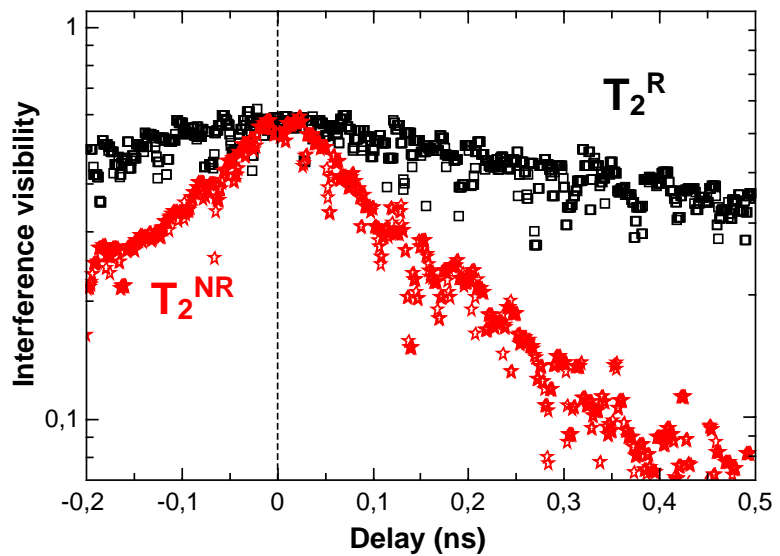

FIG. 2: Fourier transform spectrum in a semi-log scale of a single QD emission line for resonant (black squares) and nonresonant (red stars) excitation. The coherence time is 0.95 ns and $0.2 \mathrm{~ns}$ for resonant and NR excitation respectively. Data provided by courtesy of L. Monniello, INSP Sorbonne University.

AlGaAs/GaAs Bragg mirrors, which is often the case to enhance the light-matter interaction, it is known that aluminium is a potential source of free charges in the material. Therefore, each QD experiences a specific electrostatic environment which will influence its emission properties in many ways through the action of an induced Coulomb field.

In early experiments, the most commonly observed feature in photoluminescence (PL) was line broadening, that was attributed to the consequent spectral wandering of the energy position of the optical transition. This was reported in single CdSe nanocrystals where an important blinking phenomenon was observed $30 / 81$. In selfassembled QDs, blinking periods of the order of seconds were reported with meV spectral shifts of the QD transition energies 15 . These large values were an indication of important electrostatic fluctuations, that have been reduced since then to a few $\mu \mathrm{eV}$ Stark shifts $37|44| 82$ thanks to the improvements in sample growth and resonant probing.

- Optical characterisation of spectral diffusion. In most of the micro-PL measurements performed on single self-assembled QDs, spectral diffusion mainly leads to a broadening of the emission line, even in the case of RF experiments. Fourier transform spectroscopy (FTS) is a common technique to measure the broadening of narrow spectral lines with a very high resolution $7 / 18|43| 53 \mid 61$. The interference contrast is related to the nature of the broadening, homogeneous or inhomogeneous, corresponding to a Lorentzian or a non-Lorentzian spectral lineshape respectively. For instance, the characteristic spectral line of an ideal TLS only coupled to an electromagnetic field is Lorentzian with a radiatively-limited broadening 39 . Then, the corresponding FT spectrum is a mono-exponential function with a characteristic decay time $\mathrm{T}_{2}=2 \mathrm{~T}_{1}$. This kind of experiments is also called "auto-correlation" measurement since it allows to estimate the first-order correlation function $g^{(1)}(\tau)$.

Therefore, FTS is a useful way to extract information on the different dephasing mechanisms and their effect on the emission line-broadening of single QDs. Indeed, early FTS measurements on QDs showed how spectral diffusion induces a modification in the shape of the

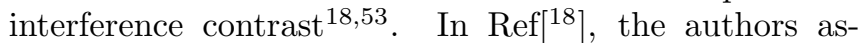
sumed that the fluctuating reservoir of carriers around the QD produces a random energy shift of the QD emission line with a modulation amplitude $\Sigma$ and on characteristic timescales $\tau_{c}$. These parameters are related to the population fluctuations and capture/escape times of trapped carriers in the localization sites. Depending on the factor $\Sigma \tau_{c} / \hbar$ two regimes of incident power were found, where a qualitative change in the contrast of the Fourier spectrum shape occurs. The profile change from a mono-exponential $\left(\Sigma \tau_{c} / \hbar<1\right)$ to a Gaussian function $\left(\Sigma \tau_{c} / \hbar>1\right)$. At low incident power, the line is Lorentzian because the changes in the reservoir configurations are fast and the fluctuations are smoothed out, while at higher pump power the modulation is slow, the line becomes Gaussian and the spectrum reflects the statistical distribution of the emission energies (see Fig 3).

This behavior was attributed to the different efficiency rates of carriers capture in, and escape out of the traps in semiconductor nanostructures at low temperature. While the capture rate is always very efficient by optical phonon-assisted emission, the escape processes are activated by raising the power, due to Auger-like processes. Then, at low power $\tau_{c}$ is short and almost constant, only governed by the capture time. $\Sigma$ is also small since the traps are almost always filled. At high power, Auger scattering becomes non negligible leading to a more noisy electrostatic environment. Then $\Sigma$ increases as more configurations for the trapped carriers become available. So, $\Sigma$ depends on the ratio of capture and escape times and increases with power. An interesting point which was noticed, is that even in the presence of spectral diffusion, at low pump power it is still possible to define a coherence time $T_{2}=\hbar^{2} / \Sigma^{2} \tau_{c}$ since the line is Lorentzian. Nevertheless, the coherence times that were found were very short, a few tens of ps, leading to quite broad linewidths of hundreds of $\mu \mathrm{eV}$, an effect that has to be attributed to the intense above-band non-resonant laser excitation which photocreates multiple free carriers inducing a noisy electrostatic environment.

With intradot laser excitation (i.e. resonant with some excited state in the dot) and with an additional aboveband non resonant laser, Kamada and coworkers ${ }^{53}$, confirmed that in the case of a QD with carriers trapped in surrounding localized states, the FT spectrum evolves from a mono-exponential to a Gaussian function with increasing above-band carriers population. On the contrary, the function remains a single exponential in the case of a very unique QD free from charges in its neigh- 

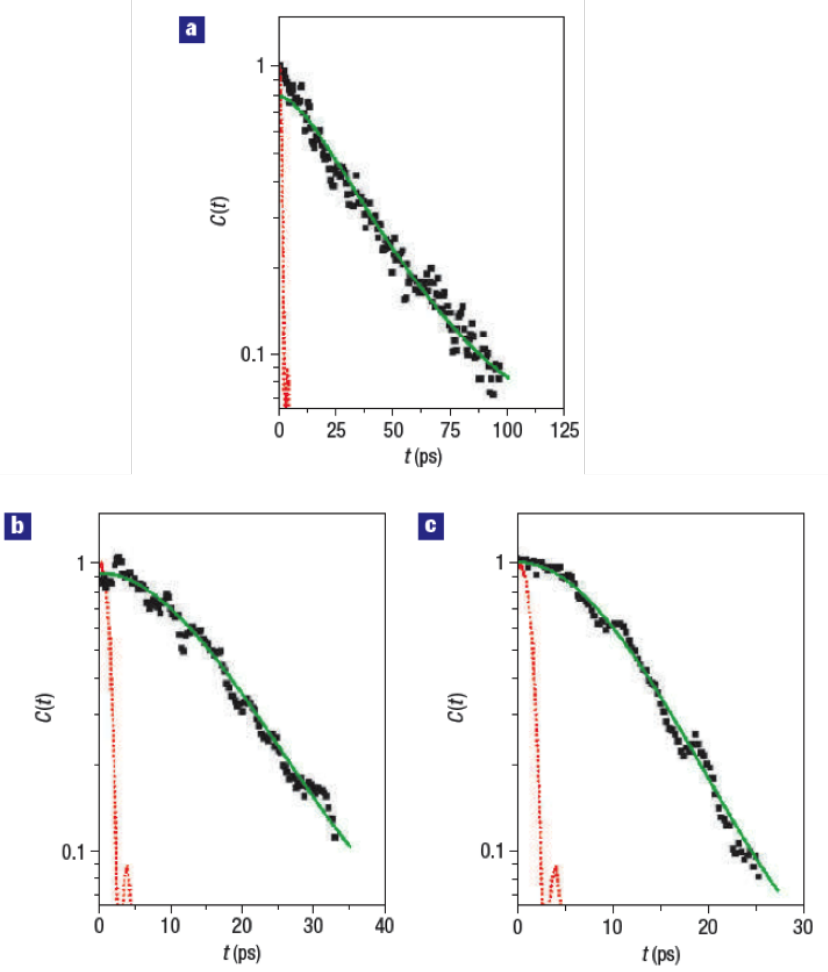

FIG. 3: (a)-(c) FT spectra of the emission line of a single QD as a function of the incident power at $10 \mathrm{~K}$. The interference contrast corresponds to a Lorentzian line at low pump power (a), and evolves to a Gaussian line when increasing the pump power (b)-(c). The dotted red line corresponds to the system response function and the solid green lines are theoretical fits with $\Sigma \tau_{c} / \hbar \sim 0.6$ (a), 1.05 (b), 1.35 (c). Figure reproduced from Ref.[18].

boring environment.

- Results under resonant excitation. When using resonant excitation, different situations have been encountered and very often the FT spectra can neither be adjusted by a single exponential function nor a Voigt profile ${ }^{112}$. This is due to the fact that the characteristic time on which spectral diffusion occurs is at least one order of magnitude longer than the radiative lifetime. Figure 4, shows interferograms obtained for three QDs having different electrostatic environments leading to different effective coherence times $\mathrm{T}_{2}$ (Fig 4(a)-(c)). In that case, the FT spectra have been adjusted using a "pseudo-Voigt" function which is defined by $f(t)=$ $(1-\eta) e^{-|t| / T_{2}}+\eta e^{-\left(t / T_{2}\right)^{2}}$. The parameter $\eta$ is related to any inhomogeneous contribution that will give rise to a Gaussian shape of the interference contrast and reflects the slight energy shift of the RF because of the fluctuating electrostatic environment. Thus, $\eta$ reveals the inhomogeneous broadening of the line due to spectral diffusion. $T_{2}$ is an effective coherence time with $\mathrm{T}_{2} \neq 2 \mathrm{~T}_{1}$. The lower $\eta$ is, the closer the FT spectrum is to a mono- exponential function and the closer the related linewidth is to a radiatively-limited broadening (see Fig 4). On the contrary, $\eta=1$ means that the inhomogeneous broadening is dominant.

Additionally, the issue of RF quenching strongly suggests that in most of the QDs either resonant absorption cannot occur or resonant emission is inhibited. In the former case, resonant absorption can be quenched because of Coulomb blockade effect and in the later case, resonantly photo-excited carriers can experience different scattering mechanisms that prevent e-h radiative recombination. These two situations will be discussed in more details below. In non-intentionally doped QD structures non-resonantly excited, the commonly encountered situation is to observe two main lines: one corresponding to a neutral e-h pair and a detuned one, corresponding to a positively or negatively charged e-h pair depending on the kind of captured carrier in the dot (see Fig 6a). Optical charging or residual doping in the sample are the main sources for the presence of free carriers that can be captured in the dot. Then, the configuration of charges in the vicinity of the dot, will influence in a different way the probability of RF quenching for a neutral or a charged e-h pair $\frac{75}{7}$. Recently, similar quenching effects in charge-tunable QD devices have been observed and attributed to the photo-generation of free electrons from the highly doped back contact, that can relax into the QD and block the emission from the neutral e-h pair 83 .

- Carrier trapping in defects. Based on the assumption that the main source of residual carriers are holes, as discussed previously, most of the following examples will be analyzed through the prism of holes trapped in defects lying close to the investigated dots. However, an analogous discussion can be made in the case of electrons. Then, the occupancy of the traps can influence in many ways the QDs optical properties. Indeed,

(i) If a nearby trap - typically several tens of nanometers away from the dot ${ }^{4484}$ - is filled, a few $\mu \mathrm{eV}$ discrete jump of the QD absorption energy can occur $\underline{44 \mid 45}$. The trapped charge induces a local electrostatic field at the origin of the quantum confined Stark effect (Fig 5a) which is pronounced because of the large polarizability of the QDs ${ }^{85}$. If the traps are shallow, the charges might go out and get trapped elsewhere, creating a fluctuating electrostatic environment that dynamically changes the energy level position leading to dephasing processes. The dynamics of this phenomenon can be probed, provided it is not limited by the time resolution of the experiment. Typical time scales of the charging/discharging mechanisms are in the microsecond range.

(ii) If the trap is close enough to the QD, carriers can tunnel in and out of the dot, and two situations must be considered to understand the quenching of the RF. If the trap is initially filled with a hole and the dot empty (Fig 5b), the hole can tunnel from the trap to the dot preventing the resonant absorption of a neutral e-h pair, due to Coulomb interaction between carriers. In the second case where the trap is initially empty (Fig 5c), resonant 


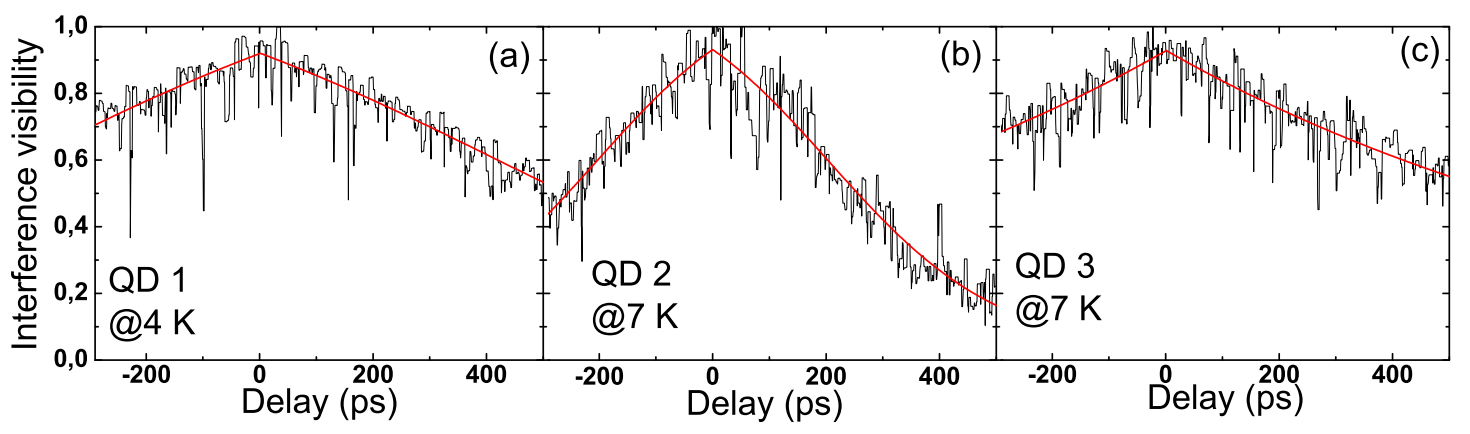

FIG. 4: (a)-(c) Fourier transform spectra for different QDs under resonant excitation with an additional weak NR He-Ne laser (a few $\mathrm{nW}$ ) to enhance the RF signal. The acquisition time is $1 \mathrm{~s}$ for each data point, which means that all the dephasing processes will be averaged within this time scale. The contrast is adjusted using a pseudo-Voigt function (see text). Values for QD1: $\mathrm{T}_{2} / \mathrm{T}_{1}=0.35 ; \eta=0.45 ; \mathrm{QD} 2: \mathrm{T}_{2} / \mathrm{T}_{1}=0.23 ; \eta=0.55 ; \mathrm{QD} 3: \mathrm{T}_{2} / \mathrm{T}_{1}=0.71 ; \eta=0.10$. Figure reproduced from Ref $\left[6_{1}\right]$.

absorption of the neutral e-h pair can take place. However, if the hole tunneling time from the dot to the trap is faster than the radiative recombination time, the electron is left in the dot and radiative recombination cannot occur.

Hence, the important parameters ruling the impact of charge noise are: the number and location of traps with respect to the position of the $\mathrm{QD}$, as well as the time scale on which the charging/discharging mechanisms occur with respect to the radiative lifetime and the measurement time. Different spectral features of the emission can be observed, like discrete spectral jumps, continuous drift or just broadening. The random capture and release of single carriers - already present in the materialin traps close to the QD have been monitored in real time with a $\mu$ s time resolution ${ }^{75 / 82}$. Although carrier capture on localisation sites is known to be fast due to phonon-assisted processes $\frac{18}{18}$, the slow charge fluctuations that are here unveiled seem to be consistent with Augerlike processes ${ }^{44 / 75 / 82 \mid 86}$. In this way the $\mathrm{QD}$ is a very sensitive probe of the local electric environment at the single charge leve $\underline{44|82| 87}$.

\section{CHARGE NOISE : AN UNAVOIDABLE EFFECT?}

\section{A. The additional non-resonant excitation}

The use of an additional above-band, non-resonant (NR) laser excitation (for instance, a He-Ne laser or a laser absorbed in the wetting-layer continuum) was proposed in order to: (i) recover the intrinsic properties of the resonant emission and (ii) help characterise more quantitatively the fluctuating electrostatic environment of the dots. Monte-Carlo simulations have been used to model the random capture and escape of carriers into the localisation sites close to the $\operatorname{dot} \sqrt{18 / 44 \mid 53}$. By simulating (a)

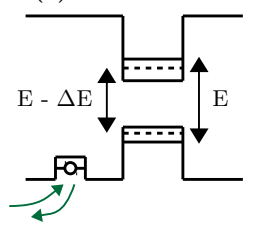

Dephasing process (b)

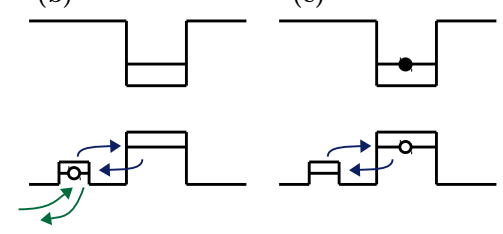

Quenching of the RF
FIG. 5: Scheme of the different trapping mechanisms of free carriers (holes in this example). (a) Defects lying close to the dot can trap freely moving carriers present in the material. A small Stark shift, $\Delta E$, is experienced by the QD electronic levels. When an e-h pair is photocreated in the dot, the intermittent occupancy of the trap leads to a discrete jumps of the optical transition causing dephasing. (b) A trapped carrier in a close-lying defect can tunnel in the dot, prohibiting absorption at the energy of the neutral e-h pair. However the $\mathrm{RF}$ of the charged pair can be observed. (c) If the defect is empty and an e-h pair is resonantly photocreated in the dot, the hole can tunnel out. The electron is left in the dot and the RF of the neutral pair is quenched. The green arrows represent the carrier capture in the defect and out of it; the blue arrows represent the tunneling in and out of the dot.

Stark shifts which can be compared to experimental results, important informations have been provided on the characteristic timescales of trapping, as well as on the number, occupation probability and relative position of the traps with respect to the dot.

- Regain of the RF. To circumvent the problems related to the fluctuating electrostatic environment it has been reported in the literature that an additional NR excitation can help to recover the $\mathrm{RF}$ 7/33/61/84. This proposition is linked to early studies reporting that an additional infrared laser tuned below the QDs ground state, can lead to a considerable change of the QDs photoluminescence signa $80[89$. This observation was interpreted as an ef- 

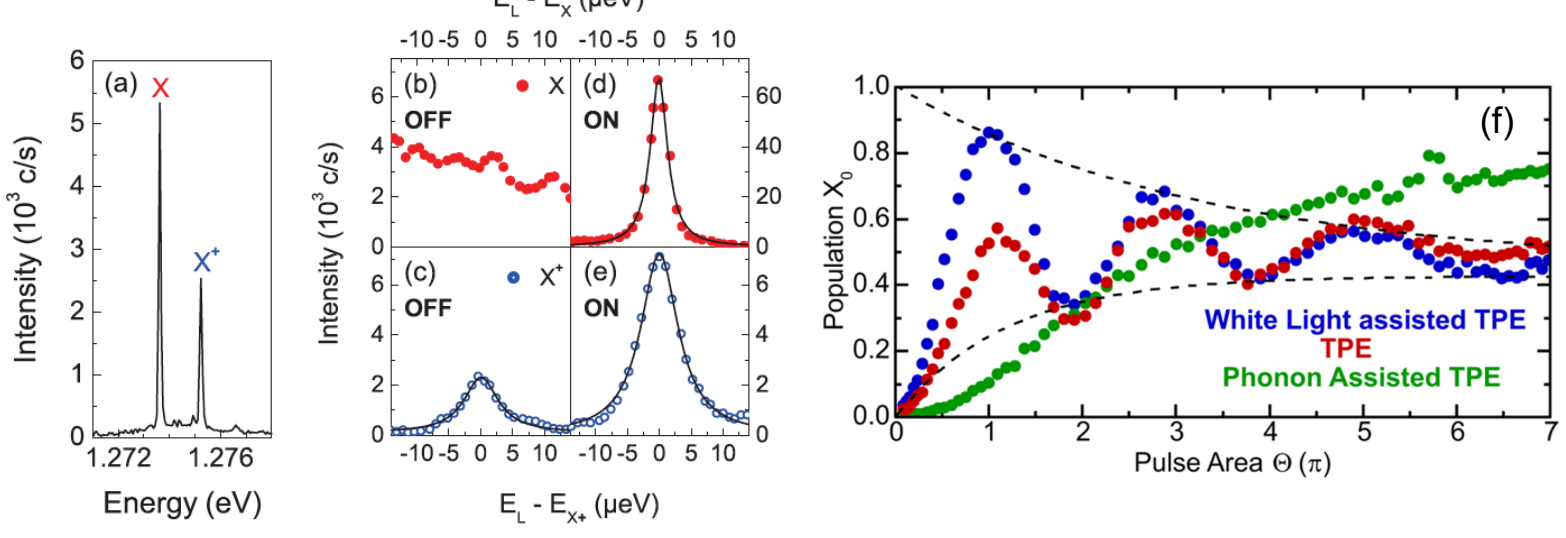

FIG. 6: (a) Typical micro-photoluminescence spectrum of a single self-assembled InAs QD excited out of resonance, showing the neutral e-h pair $(\mathrm{X})$ and the positively charged trio $\left(X^{+}\right)$. (b)-(e) Resonant emission spectra of $\mathrm{X}$ and $X^{+}$, as a function of the detuning of the laser, with $(\mathrm{ON})$ and without $(\mathrm{OFF})$ the additional NR He-Ne laser. Figure reproduced from Ref 75 . (f) Rabi oscillation of the neutral e-h pair population (labelled $X^{0}$ ) in a resonant two-photon excitation scheme with (blue) and without (red) white light. Figure reproduced from Ref 88.

fective separate generation of free electrons and holes in the sample through the excitation of deep level defects present in the band gap of the barriers. The excess carriers can either screen the electrostatic field or neutralize the different charge traps, inducing significative increase of the PL intensity of single QDs.

The above-band laser in addition to the resonant optical excitation creates a similar effect. In RF experiments, the use of a NR He-Ne laser at very low excitation power - so it does not generate luminescence in the absence of the resonant laser - photocreates carriers that can be captured separately in the dot (either an electron or a hole) on a microsecond scale, helping the resident charge in the dot to recombine radiatively ${ }^{33}$ (see Fig 5 b). Indeed, the QD ground state which is occupied by one charge (a hole in the case of Ref [33]), empties, and absorption of one photon resonant with the neutral e-h pair transition can occur. The resonant photogeneration of an e-h pair in the dot is very fast, compared to the slow characteristic tunneling time into the dot which is of the order of hundreds of microseconds $\sqrt{33}$. Therefore, the increase of the $\mathrm{RF}$ intensity under the additional NR laser excitation, depends only on the intra-dot charge occupancy. Many groups have reported an increased brightness of the RF that can reach one order of magnitude $\mathrm{e}^{7|33| 61|74| 84 \mid 90}$.

Figure 6 illustrates the typical situations of RF quenching and regain described above. When the NR He-Ne laser is off (Fig 6b), the optical transition of the neutral e-h pair is quenched, while an intensity recovery is observed with the He-Ne laser on (Fig 6d). On the contrary, the RF of the charged e-h pair is always present (Fig 6c), with an enhancement of the intensity signal observed with the NR laser turned on (Fig 6e). A scenario with two holes resident in the QD was suggested to explain this results 55 .
Resonant Rabi oscillations experiments performed on GaAs/AlGaAs droplet QDs ${ }^{88}$ with an additional weak white-light source induced an increase in the RO amplitude. In a two-photon excitation scheme with $\pi$-pulses, the maximum population probability was enhanced by almost a factor of two as shown in Fig 6(f). This behavior is QD dependent and attributed to a stabilization of the electric field experienced by the QD, combined to a suppression of other non-radiative recombination processes.

The presence of an additional above-band excitation can also screen the local electric field in the immediate environment of the dots, inducing an energy shift of the $\mathrm{RF}$ or the absorption spectrum due to the quantum confined Stark effect ${ }^{44 / 75 / 82}$. This is illustrated in Fig 7a-b, which represent the energy shift of the resonant emission of the neutral and positively charged e-h pair as a function of the NR additional laser power. A shift of a few $\mu \mathrm{eV}$ is observed, attributed to the electric field created by holes trapped in the vicinity of the dot. The observed red (blue) shift was attributed to a decrease (increase) in the number of holes according to rate equations taking into account all the capture and escape processes induced by the optical gate $e^{75}$.

Figure $7 \mathrm{c}$, shows laser spectroscopy results with and without an additional NR $830 \mathrm{~nm}$ laser where the differential reflectivity is measured. The QDs are embedded in a n-i Schottky diode, so when the NR laser is turned on, the resonance shifts due to the Stark effect and a negative bias has to be applied to the diode to recover the resonance. We will show in the next paragraph how the location of the defects with respect to the dot position could be extracted from these measurements.

In terms of line broadening, the additional NR laser leads to narrower RF lines when the defect occupation is 
(a)

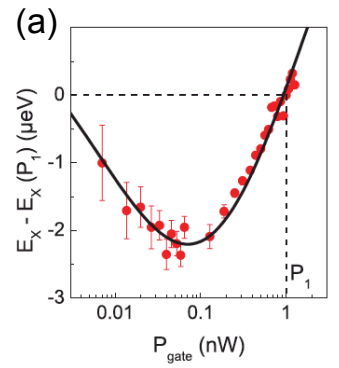

(b)
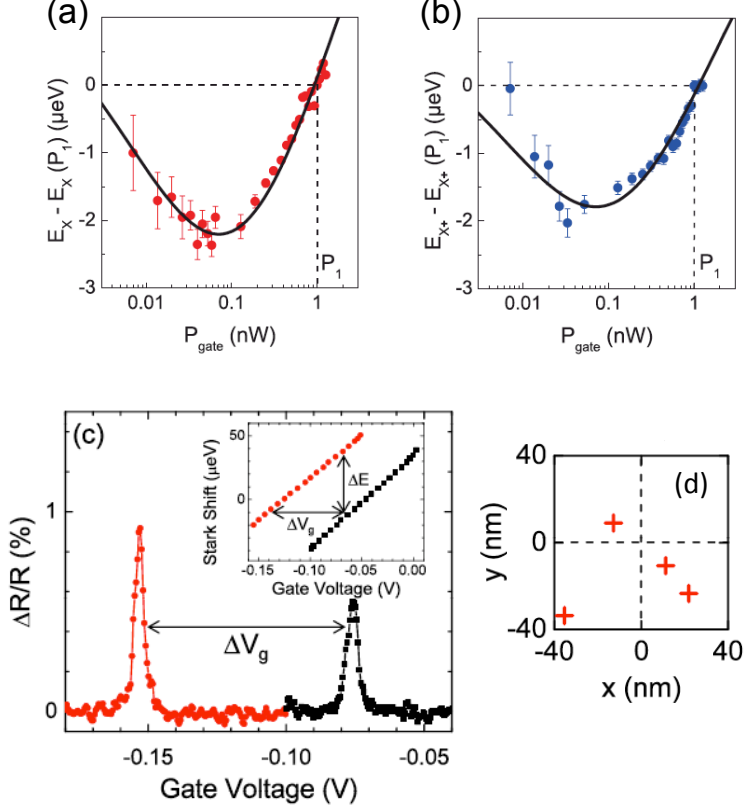

FIG. 7: Illustrations of the quantum confined Stark effect in presence of an additional NR laser. (a) Energy of the neutral e-h pair and positively charged pair (b) resonant emission as a function of the NR laser power, denoted $P_{\text {gate }}$. (a)-(b) Figure reproduced from Ref [75]. (c) Differential reflectivity measurements for a QD versus external gate voltage for constant resonant laser wavelength, without (black) and with (red) a NR laser. (d) Lateral location of localization sites with dot position at the center. The plane of the localization sites lies above the QDs at the distance separating the QDs plane from the capping layer. (c)-(d) Figures reproduced from Ref [44].

frozen 33144 . The linewidth decreases exponentially with He-Ne power ${ }^{90}$ and gets close to the radiative limit but the RF line shape can still be a Gaussian, suggesting the presence of some remaining spectral wandering.

We will discuss in the next section the frequency dependent read-out of the RF since it gives valuable information about the characteristic time scales on which charge noise occurs. So, if the RF is probed on a short enough time scale, it is possible to measure close to transform-limited RF lines.

- Characteristics of the local electrostatic environment. Additionally, the use of a NR excitation gave precious indications about the local charge environment and helped to characterize the trapping/detrapping time of carriers in the defects, or the spatial separation between charged traps and dot 4484 . Chen et at ${ }^{84}$, performed resonant photoluminescence excitation spectroscopy using both a very narrow tunable laser and an additional above-band excitation which allowed to unveil the timeaveraged charge occupation probability of the traps and the induced Stark shifts of the RF lines. In that case, the probed QD had two nearby traps that have been evidenced with zero or one charge level occupation, giving rise to four possible configurations. Free carriers are photocreated by the NR excitation and the traps are assumed to be empty in their initial configuration. Performing second-order correlations measurements as a function of the NR excitation power, the authors were able to extract values of the characteristic time corresponding to effects of charging dynamics both in the traps and the $\mathrm{QD}$, ranging from $1 \mathrm{~ms}$ to tens of $\mu \mathrm{s}$. Unfortunately, in their measurements they could not deduce independently the contribution of each effect. These results are consistent with those obtained by Nguyen et al ${ }^{75}$ who found typically one hundred $\mu$ s for the trap charging dynamics.

In specifically designed charge-controlled devices, Houel et $a{ }^{\sqrt{44}}$ performed differential reflectivity measurements with an additional low-power NR excitation, combined to Monte-Carlo simulations to estimate the location of the defects with respect to the QD. The differential reflectivity measurements showed discrete energy jumps from a few tens of $\mu \mathrm{eV}$ that the authors attributed to single-charge fluctuations in the defects (Fig 7c). This induces a difference in the local electric field at the position of the dot which in turn induces the observed Stark shift that was compared to Monte-Carlo simulations. The distance of defects lying above the dots was found to be within a hundred of nanometers away from the QD, located at the heterointerface between the GaAs capping layer and the short period AlAs/GaAs superlattice grown on top of it. This is illustrated in Fg $7 \mathrm{~d}$, where the red crosses represent the lateral location of the defects with respect to the central position of the dot. In the case where the defects are in the same plane as the dots, the component of the electric field cannot be estimated but a smaller Stark shift of a few $\mu \mathrm{eV}$ is expected.

- Some peculiar features. Despite the interesting results regarding charge noise characterization and regain of the RF, the use of an additional NR laser can be very tricky. Indeed, some properties of the QD emission can be modified in a way that is not so trivial to understand. For instance, Fig 8 illustrates a situation where for some specific QD, the NR laser helps to recover the RF of the neutral pair (Fig 8a), while it quenches the RF in another one (Fig 8b), the behavior of each line depending on the initial charge configuration in the dot and around it. This observation which is opposite to the situation presented in Fig 6a, suggests once more that the charge noise is sample dependent, and even dot dependent.

Moreover, the additional NR laser can strongly impact the QD Rabi oscillations ( $\mathrm{RO}$ ) that can also be modified in a non-trivial way. Figure $8(\mathrm{c})$, shows RO of a given $\mathrm{QD}$ under three different He-Ne laser powers. The RO period seems to increase with the power of the He-Ne and is four times longer when the power is multiplied by a factor of ten. A tentative qualitative interpretation could be the build-up of a static electric field around the $\mathrm{QD}$ due to the photocreated charges by the He-Ne laser, which reduces the oscillator strength of the e-h pair in the dot thus increasing the period of the RO. 

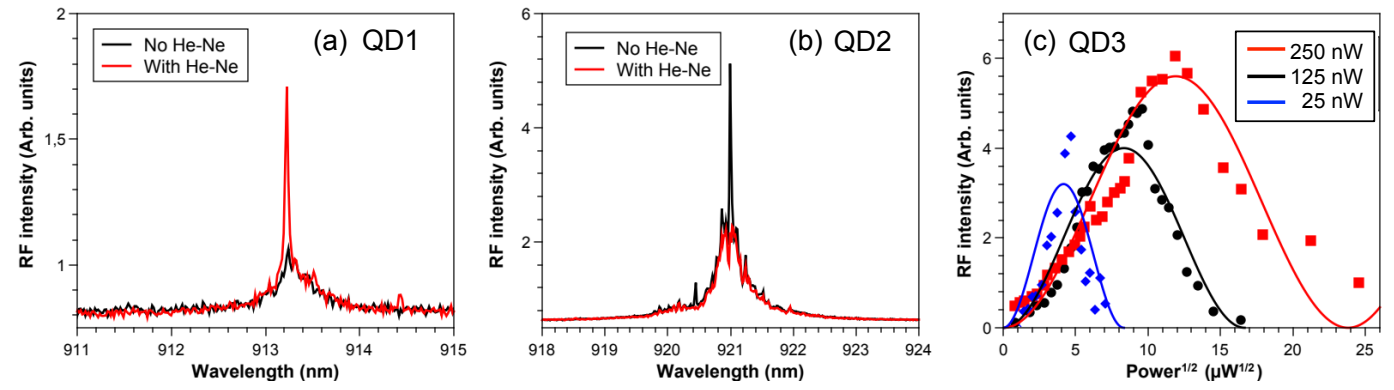

FIG. 8: (a)-(b) Low temperature RF spectra for two different InAs/GaAs QDs excited with $\pi$-pulses. The background signal is due to scattered laser by the one-dimensional waveguide in which the QDs layer in embedded. The red (black) curve is obtained with (without) the presence of a very weak above-band He-Ne laser. (c) Rabi oscillations on another QD in the same sample, in presence of an additional He-Ne laser at different powers of $25 \mathrm{nW}, 125 \mathrm{nW}$ and $250 \mathrm{nW}$, corresponding to the blue, red and black curve respectively. (a-c) Data provided by courtesy of C. Tonin, INSP Sorbonne University.

\section{B. Charge-controlled devices}

As we have seen previously, the charging of QDs in a sample without a diode structure, is completely uncontrolled and occurs either by capture of a charge from the environment (see Fig. 5), or by optical charging when an above-band excitation is used - i.e. when an e-h pair is photocreated and one carrier is captured separately in the QD. However, for spin-based qubits it is highly desirable to control the charge occupancy inside a QD using a charge-controlled device, that will also potentially allow to control and freeze the electrostatic environment in the vicinity of the dots. By designing suitable gated devices it is possible to observe single, clear lines of one neutral e-h pair, one charged pair, two neutral pairs, etc, showing that it is possible to control the charge of the $\mathrm{QD}$, carrier by carrie ${ }^{91} 95$. Within the voltage plateaus where these lines are observed, the charging/discharging mechanisms from the nearby traps are disabled and the e-h complexes are stabilized in the dot. Resonant experiments then give rise systematically and without any quenching, to very intense luminescence lines with in-

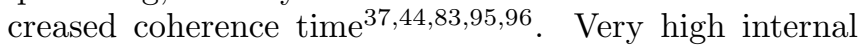
emission efficiency of single photons close to unity is also reached ${ }^{95}$. Thus, a voltage-controlled device neutralizes quite successfully the electrostatic environment without the use of an additional NR laser and allows achieving single photon sources with high performances ${ }^{97}$.

In gated ridges structure for resonant excitation ${ }^{95}$ (Fig 9a-b), it is possible to address on resonance different optical transitions, like the single negatively charged trio. For instance, RF experiments (Fig 9c) have been performed with $\pi$-pulse excitation on the negatively charged trio line (labelled $\mathrm{X}^{-}$) and showed that the coherence time $\mathrm{T}_{2}$ measured by FTS is increased in the voltage range where the trio is stabilised ${ }^{95}$. Figure $9 \mathrm{~d}$ represents the $\mathrm{T}_{2}$ measurements for different values of applied bias, showing clearly how the coherence time is constant in a given voltage range. However the measured values of $\mathrm{T}_{2}$ correspond to a broadening which is still twice the radiative limit $(1.2 \mu \mathrm{eV}$ while the radiative limit is $0.5 \mu \mathrm{eV}$ in this particular QD). The origin of the broadening is inhomogeneous, as deduced from the analysis of the FT spectra and the high values of the $\eta$ parameter (Fig 9e) discussed in section III.

Since the contribution of the phonon bath to the line broadening is negligible ${ }^{4198}$, the remaining dephasing could be attributed to spin noise which is likely important for negatively charged complexes, because of the unpaired electron ${ }^{37}$. Residual charge noise cannot be excluded though. It is worth noticing that close to the thresholds of stabilization of the $\mathrm{X}^{-}$trio, there is an important coupling to the Fermi reservoir 91991100 . At these bias thresholds, electron tunneling from (into) the reservoir into (from) the dot, is favored and becomes dominant compared to spectral diffusion, resulting in a drop of the coherence time. A similar effect has been observed in measurements of the optical linewidth of the neutral e-h pair 101. An abrupt increase at the edges of the Coulomb blockade plateau was shown, related to a fast electron spin dephasing due to co-tunneling with the Fermi sea. A strong interaction with the Fermi sea can even lead to hybridized states between the negatively charged trio and the reservoir continuum concomitant with a broadening of the optical linewidth 99102 .

Surprisingly, at the two thresholds labelled $U_{1}$ and $U_{2}^{\prime}$ (Fig 9d), the variation of $T_{2}$ is not similar. This effect has been attributed to different bias-induced tunneling dynamics at the two thresholds. Close to $U_{2}^{\prime}$, it is the excited state of the dot (one photocreated e-h pair plus a single electron) that interacts with the electron reservoir on a large bias range. Within this range, tunneling between the Fermi sea and the second conduction level occurs. On the contrary, close to $U_{1}$, it is the ground state (empty dot) that interacts with the electron reservoir on a narrower bias range, since tunneling occurs between the Fermi sea and the first conduction level. Then, the intensity of the RF signal drops abruptly close to $U_{1}$ and it becomes difficult to measure the coherence time. These differences in the electron tunneling dynamics from the 

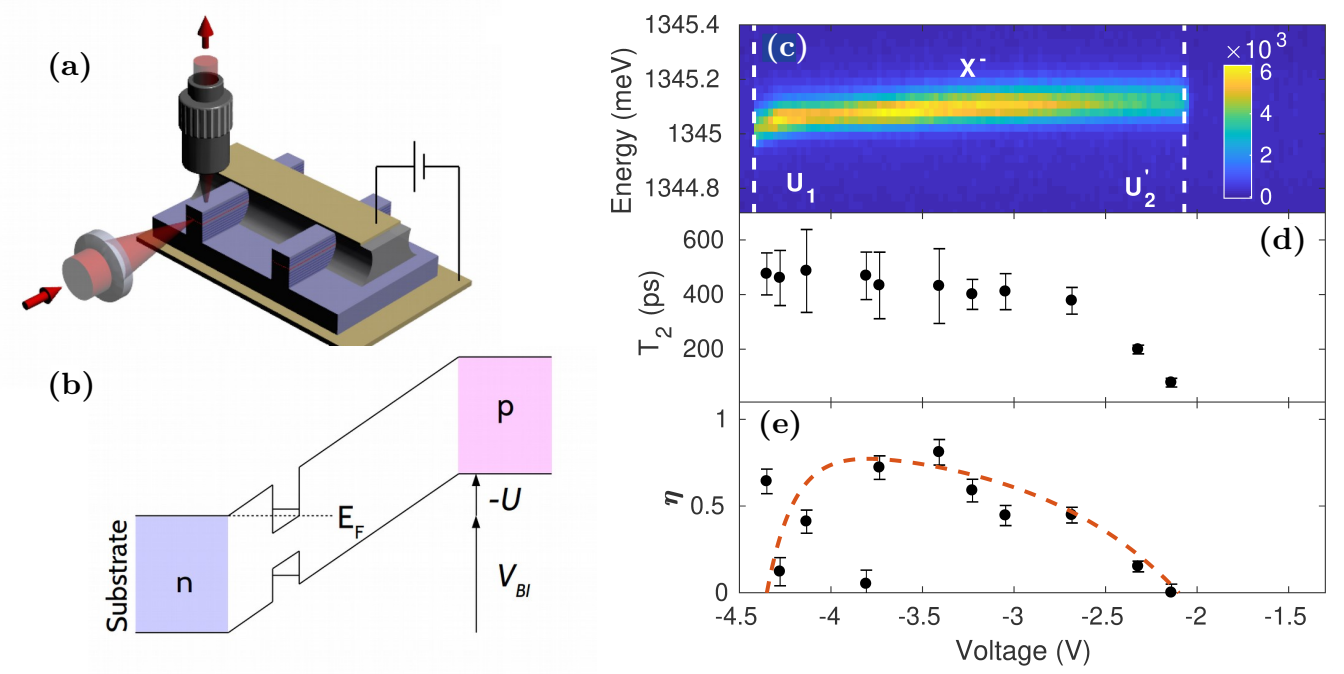

FIG. 9: Schematic representation of (a) the voltage-controlled one-dimensional waveguide sample for RF experiments and (b) the simplified band structure. (c) RF map of the negatively charged trio line (labelled $\mathrm{X}^{-}$) as a function of the gate voltage. The color plot is in units of counts/s. The voltages $U_{1}$ and $U_{2}^{\prime}$ correspond to the thresholds where the trio is stabilized in the dot. (d) Coherence time $T_{2}$ measured by FTS within the voltage range of the existence of the trio. (e) Values of the parameter $\eta$ which characterizes the inhomogeneous contribution of the pseudo-Voigt profile used to analyse the FT spectra. Adapted from $\operatorname{Ref}\left[\frac{95}{[}\right]$.

Fermi reservoir to the dot have also been discussed by Kurzmann et al. in [79].

\section{Regarding coherence properties}

As pointed out previously, the timescale on which spectral diffusion occurs has to be compared to the radiative lifetime of the QD but also to the characteristic timescale of the measurement.

Indeed, in high purity gated-samples with very low concentration of residual impurities 37 , by deriving noise spectra from the Fourier transformation of RF time traces (Fig 10a), the nature of noise could be identified. The usual two main sources of dephasing come into play: (i) charge noise, due to the fluctuating electrostatic field, affects predominantly the neutral pair $X^{0}$; and (ii) spin noise, due to the fluctuating nuclear magnetic field perturbs mostly the negatively charged trio $X^{-}$because of the unpaired electron. Since the local electric field fluctuates in time, a dc Stark shift of the optical resonance is induced with respect to the laser which is fixed in the experiment. The noise spectra as a function of detuning between the laser and the energy position of the line are different for the two dephasing processes and allow to distinguish charge noise from spin noise (Fig 10a). Indeed, charge noise results in a rigid shift of the optical resonance leading to a small change in $\mathrm{RF}$ for zero detuning and a large change at $\delta=\Gamma / 2$. On the opposite, spin noise induces a Zeeman splitting in the resonance, resulting in a large change in $\mathrm{RF}$ at $\delta=0$ and a small change in $\mathrm{RF}$ at $\delta=\Gamma / 2$.

Another crucial information is that the influence of the fluctuating electrostatic environment is dominant at low frequencies, while spin noise is dominant at high frequencies. Above $50 \mathrm{kHz}$, the combined charge and spin noise drops rapidly. The RF linewidth was then measured as a function of the scanning frequency defined as the derivative of the detuning normalized by the radiatively-limited linewidth 37 . Nearly radiative-limited lines were achieved when the RF was driven at high frequency around 50 $\mathrm{kHz}$. It means that spectral diffusion occurs on characteristic timescales slower than tens of $\mu \mathrm{s}$. It is a much slower process than radiative recombination, which is of the order of one ns, i.e. in the $\mathrm{GHz}$ range. Further improvements allowed to reach radiatively-limited RF lines on both the $X^{0}$ and the $X^{-}$lines by controlling the nuclear spins with optical excitation, even if the measurements were recordered on a timescale of one second 101 .

In the light of the above mentioned results, we can now understand why in the FT spectra the inhomogeneous broadening can be important in standard samples and experiments. Indeed, the usual integration time of these measurements is long enough (timescale of seconds) to average charge noise effects. This leads to a reduced coherence time $T_{2}<2 T_{1}$ and linewidths which are not transform-limited. On the contrary, when the device is operated fast enough, the effect of charge noise is frozen 
(a)

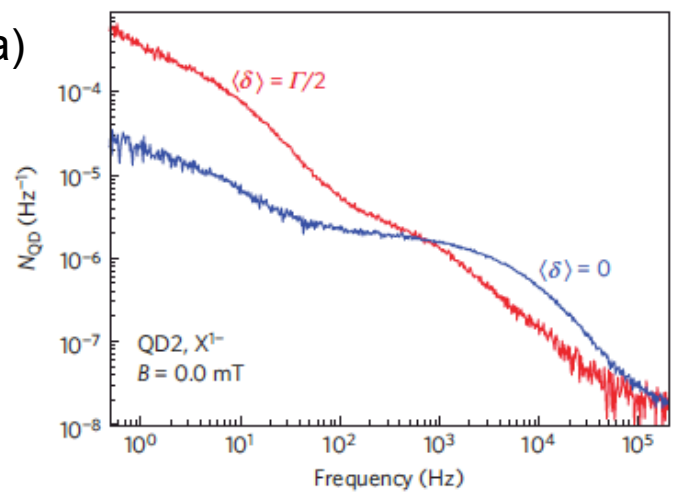

(b)

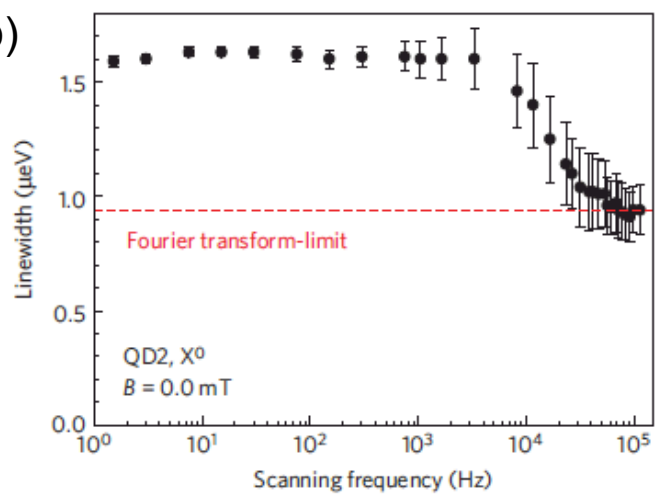

FIG. 10: (a) RF noise spectra for a negatively charged trio of a QD (labelled QD2) at low temperature and zero magnetic field. The noise spectra were obtained by Fourier transforming the time traces of the RF. The two curves were recorded at two different averaged detunings with respect to the wavelength of the laser: blue for $\langle\delta\rangle=0$ and red for $\langle\delta\rangle=\Gamma / 2$, with $\Gamma$ the full width at half maximum of the RF line. The noise at low frequency is shown to originate from charge noise while at high frequency it originates from spin noise. (b) $\mathrm{RF}$ linewidth versus the scanning frequency. The dashed red line shows the radiatively limited broadening corresponding to twice the radiative lifetime $\left(\mathrm{T}_{1}=700 \mathrm{ps}\right)$. Figure reproduced from Ref [37].

and the radiative limit can be reached.

This has important consequences on the degree of coherence of the emitted photons. In Hong-Ou-Mandel experiments 103 where the indistinguishability of photons is measured, very high degrees of indistinguishability of up to $95 \%$ were found $861 \mid 104$. This was surprising because it was expected that the dephasing processes due to interaction of the QD with the environment should influence the coherence properties and reduce the indistinguishability. Actually, what matters is the characteristic timescale of the HOM experiment determined only by the time delay between the excitation pulses, typically a few ns. At this timescale, only the interaction with the phonon bath is relevant ${ }^{61}$ and thus, the effect of charge noise does not influence the indistinguishability. This was confirmed in an experiment ${ }^{105}$ where the two- photon interference was measured as a function of the time delay between the emitted photons; the degree of indistinguishability drops from $96 \%$ to $92 \%$ as the emission time increases from $1 \mathrm{~ns}$ to $15 \mu \mathrm{s}$. Delbeq et al ${ }^{106}$, showed in gated defined QDs, that the photons' coherence time emitted from the QD decreases with the measurement acquisition time showing that the value of the coherence time depends on the way we measure it.

\section{SUMMARY AND OUTLOOK}

The quest for using solid-based building blocks for quantum technology applications has driven research efforts to develop high-quality materials and master their physical properties. Epitaxial self-assembled QDs, like the InAs/GaAs systems have dominated the world of dots in the past decades, thanks to their convenient bottomup Stranski-Krastanov growth mode, their highly confined energy spectrum and the impressive achievements in nanofabrication techniques.

Despite the high purity of crystalline samples available today, it will probably be very difficult to completely remove the presence of impurities and structural defects present at the heterointerfaces of the structures. These defects create localization sites that can trap efficiently the free carriers. They are considered as an important source of charge noise that limit the coherence properties of the probed QD states. Typical distances of these defects with respect to the position of the dots layer range between a few tens to a few hundreds of nanometers. The trapped carriers create an electrostatic landscape that fluctuates spatially and dynamically in the $\mu \mathrm{s}$ range, longer than the radiative recombination time. The resulting spectral diffusion leads to broadening of the luminescence lines which are not radiatively limited, thus reducing the coherence time $T_{2}$.

More dramatic can be the situation where a carrier tunnels in and out of the dot, preventing resonant absorption or leading to inhibition of the RF. Hence, a $\mathrm{QD}$ is a very sensitive probe of the local electrostatic environment, detecting very low levels of charge noise leading to interesting applications for ultra-sensitive electrometers 4487 .

To circumvent the issue of charge noise, the use of an additional above-band optical excitation together with the resonant laser partially solve the problem of quenching and recover the RF. However, this is not a convenient solution for addressing the quantum states in a deterministic way as required for applications. Indeed, for an optical coherent control of spin qubits and efficient spin-photon interfaces, resonant excitation and chargecontrolled devices are two necessary ingredients for implementing quantum information protocols 58159 . Gatedstructures solved the problem of RF quenching. They can potentially stabilize the electrostatic environment in the vicinity of the dots and reduce the charge noise but only in a few cases of very high purity samples, radiatively- 
limited RF lines could be reached $37 / 101$

For on-demand single and indistinguishable photon sources applications, the best strategy is to embed the QDs in suitable photonic structures and take advantage of CQED effects107. Micro-pillars 67/97, and especially photonic crystal cavities 108 can greatly enhance the light-matter coupling thanks to their small volume and large quality factors, thus significantly reducing the radiative lifetime $T_{1}$ by a factor higher than 10 . In that case, even in the presence of residual charge noise which occurs on much longer timescales, extraction of photons is very fast - typically less than a hundred of ps - and combined to a high repetition rate, this device leads to almost $100 \%$ of indistinguishability. Therefore, resonant excitation and charge-controlled QDs in optical microcavities could be the successful combination for on-chip quantum nanophotonic devices, at the expense of the more advanced nanofabrication techniques required to limit the impact of etched surfaces charge states 109 .

\section{A. Acknowledgments}

The authors would like to thank Laura Thevenard and Paola Atkinson for helpful comments and discussions. voliotis@insp.jussieu.fr

1 P. Michler, ed., Quantum Dots for Quantum Information Technologies (Springer, 2017).

2 For a recent review see, Lodahl, P., S. Mahmoodian, and S. Stobbe, Rev. Mod. Phys. 87, 347 (2015).

${ }^{3}$ K. H. Madsen, S. Ates, J. Liu, A. Javadi, S. M. Albrecht, I. Yeo, S. Stobbe, and P. Lodahl, Phys. Rev. B 90, 155303 (2014).

4 O. Gazzano, S. Michaelis de Vasconcellos, C. Arnold, A. Nowak, E. Galopin, I. Sagnes, L. Lanco, A. Lemaître, and P. Senellart, Nat. Comm. 4, 1425 (2013).

5 J. Claudon, J. Bleuse, N. S. Malik, M. Bazin, P. Jaffrennou, N. Gregersen, C. Sauvan, P. Lalanne, and J.-M. Gérard, Nature Photonics 4, 174 (2010).

${ }^{6}$ M. E. Reimer, G. Bulgarini, N. Akopian, M. Hocevar, M. B. Bavinck, M. A. Verheijen, E. P. A. M. Bakkers, L. P. Kouwenhoven, and V. Zwiller, Nature Communications 3, 737 (2012).

7 L. Monniello, A. Reigue, R. Hostein, A. Lemaitre, A. Martinez, R. Grousson, and V. Voliotis, Phys. Rev. B 90, 041303(R) (2014).

8 N. Somaschi, V. Giesz, L. De Santis, J. C. Loredo, M. P. Almeida, G. Hornecker, S. L. Portalupi, T. Grange, C. Antón, J. Demory, C. Gómez, I. Sagnes, N. D. Lanzillotti-Kimura, A. Lemaítre, A. Auffeves, A. G. White, L. Lanco, and P. Senellart, Nat. Photon. 10, 340 (2016).

9 Y.-M. He, Y. He, Y.-J. Wei, D. Wu, M. Atatüre, C. Schneider, S.Höfling, M. Kamp, C.-Y. Lu, and J.-W. Pan, Nat. Nanotechnol. 8, 213 (2013).

10 X. Ding, Y. He, Z.-C. Duan, N. Gregersen, M.-C. Chen, S. Unsleber, S. Maier, C. Schneider, M. Kamp, S. Höfling, C.-Y. Lu, and J.-W. Pan, Phys. Rev. Lett. 116, 020401 (2016).

11 T. M. Kerr, C. E. C. Wood, S. M. Newstead, and J. D. Wilcox, J. Appl. Phys. 65, 2673 (1989).

12 C. Walther, J. Bollmann, H. Kissel, H. Kirmse, W. Neumann, and W. T. Masselink, Applied Physics Letters 76, 2916 (2000), https://doi.org/10.1063/1.126516.

${ }^{13}$ S. Mazzucato, D. Nardin, M. Capizzi, A. Polimeni, A. Frova, L. Seravalli, and S. Franchi, Materials Science and Engineering: C 25, 830 (2005).

14 A. Chahboun, M. I. Vasilevskiy, N. V. Baidus, A. Cavaco, N. A. Sobolev, M. C. Carmo, E. Alves, and B. N.
Zvonkov, Journal of Applied Physics 103, 083548 (2008) https://doi.org/10.1063/1.2913179.

15 H. D. Robinson and B. B. Goldberg, Phys. Rev. B 61, R5086 (2000).

16 M. Abbarchi, F. Troiani, C. Mastrandrea, G. Goldoni, T. Kuroda, T. Mano, K. Sakoda, N. Koguchi, S. Sanguinetti, A. Vinattieri, and M. Gurioli, Applied Physics Letters 93, 162101 (2008).

17 J. Seufert, R. Weigand, G. Bacher, T. Kümmell, A. Forchel, K. Leonardi, and D. Hommel, Appl. Phys Lett 76, 1872 (2000).

18 A. Berthelot, I. Favero, G. Cassabois, C. Voisin, C. Delalande, P. Roussignol, R. Ferreira, and J. M. Gérard, Nat. Phys. 2, 759 (2006).

19 M. Sugisaki, H.-W. Ren, K. Nishi, and Y. Masumoto, Phys. Rev. Lett. 86, 4883 (2001)

20 L. Besombes, K. Kheng, L. Marsal, and H. Mariette, Phys.Rev. B 65, 121314R (2002).

21 A. Majumdar, E. D. Kim, and J. Vuckovic, Phys.Rev. B 84, 195304 (2011).

${ }^{22}$ G. Sallen, A. Tribu, T. Aichele, R. André, L. Besombes, C. Bougerol, M. Richard, S. Tatarenko, K. Kheng, and J. P. Poizat, Nature Photonics 4, 696 (2010).

23 E. Schöll, L. Hanschke, L. Schweickert, K. D. Zeuner, M. Reindl, S. F. Covre da Silva, T. Lettner, R. Trotta, J. J. Finley, K. Müller, A. Rastelli, V. Zwiller, and K. D. Jöns, Nano Letters 19, 2404 (2019).

24 S. A. Empedocles, D. J. Norris, and M. G. Bawendi, Phys. Rev. Lett. 77, 3873 (1996)

25 A. L. Efros and D. J. Nesbitt, Nature Nanotechnol. 11, 661 (2016).

${ }^{26}$ V. Türck, S. Rodt, O. Stier, R. Heitz, R. Engelhardt, U. W. Pohl, D. Bimberg, and R. Steingrüber, Phys. Rev. B 61, 9944 (2000)

W. Ambrose and W. Moerner, Nature 349, 225 (1991).

28 A. Zumbusch, L. Fleury, R. Brown, J. Bernard, and M. Orrit, Phys. Rev.Lett. 70, 3584 (1993).

29 J. Wolters, N. Sadzak, A. W. Schell, T. Schröder, and O. Benson, Phys. Rev. Lett. 110, 027401 (2013)

30 X. Brokmann, J.-P. Hermier, G. Messin, P. Desbiolles, J.P. Bouchaud, and M. Dahan, Phys. Rev. Lett. 90, 120601 (2003).

${ }^{31}$ A. Muller, E. B. Flagg, P. Bianucci, X. Y. Wang, D. G. Deppe, W. Ma, J. Zhang, G. J. Salamo, M. Xiao, and 
C. K. Shih, Phys. Rev. Lett. 99, 187402 (2007)

32 R. Melet, V. Voliotis, A. Enderlin, D. Roditchev, X. L. Wang, T. Guillet, and R. Grousson, Phys. Rev. B 78, 073301 (2008)

33 H. S. Nguyen, G. Sallen, C. Voisin, P. Roussignol, C. Diederichs, and G. Cassabois, Phys. Rev. Lett 108, 057401 (2012).

34 P. Atkinson, E. Zallo, and O. Schmidt, J. Appl. Phys. 112, 054303 (2012)

5 M. Gurioli, Z. Wang, A. Rastelli, T. Kuroda, and S. Sanguinetti, Nature Materials (2019), 10.1038/s41563-0190355-y

${ }^{36}$ C. Heyn, M. Zocher, L. Pudewill, H. Runge, A. Küster, and W. Hansen, Journal of Applied Physics 121, 044306 (2017)

A. Kuhlmann, J. Houel, A. Ludwig, L. Greuter, D. Reuter, A. Wieck, M. Poggio, and R. Warburton, Nat. Phys. 9, 570 (2013).

38 P. Michler, ed., Single Semiconductor Quantum dots (Springer, 2007).

39 C. Cohen-Tannoudji, J. Dupont-Roc, and G. Grynberg, Atom-Photon Interactions: Basic Processes and Applications (Wiley, New York, 1992).

${ }^{40}$ H. P. Breuer and F. Petruccione, The theory of open quantum systems (Oxford University Press, 2010).

${ }^{41}$ J. Iles-Smith, D. P. S. McCutcheon, A. Nazir, and J. Mørk, Nat. Photonics 11, 521 (2017).

42 P.-F. Braun, X. Marie, L. Lombez, B. Urbaszek, T. Amand, P. Renucci, V. Kalevich, K. Kavokin, O. Krebs, P. Voisin, et al., Physical Review Letters 94, 116601 (2005).

43 C. Kammerer, C. Voisin, G. Cassabois, C. Delalande, P. Roussignol, F. Klopf, J. P. Reithmaier, A. Forchel, and J. M. Gérard, Phys. Rev. B 66, 041306 (2002)

44 J. Houel, A. V. Kuhlmann, L. Greuter, F. Xue, M. Poggio, B. D. Gerardot, P. A. Dalgarno, A. Badolato, P. M. Petroff, A. Ludwig, D. Reuter, A. D. Wieck, and R. J. Warburton, Phys. Rev. Lett. 108, 107401 (2012)

45 M. Hauck, F. Seilmeier, S. E. Beavan, A. Badolato, P. M. Petroff, and A. Högele, Phys. Rev. B 90, 235306 (2014).

46 T. Takagahara, Phys. Rev. B 117, 514 (1999).

47 B. Krummheuer, V. M. Axt, and T. Kuhn, Phys. Rev. B 65, $195313(2002)$

48 A. V. Uskov, I. Magnusdottir, B. Tromborg, J. Mork, and R. Lang, Applied Physics Letters 79, 1679 (2001).

49 P. Borri, W. Langbein, S. Schneider, U. Woggon, R. L. Sellin, D. Ouyang, and D. Bimberg, Phys. Rev. Lett. 87, 157401 (2001)

W. Langbein, P. Borri, U. Woggon, V. Stavarache, D. Reuter, and A. D. Wieck, Phys. Rev. B 70, 033301 (2004).

${ }^{51}$ D. Birkedal, K. Leosson, and J. M. Hvam, Phys. Rev. Lett. 87, 227401 (2001)

52 C. Kammerer, G. Cassabois, C. Voisin, M. Perrin, C. Delalande, P. Roussignol, and J. M. Gérard, Applied Physics Letters 81, 2737 (2002), https://doi.org/10.1063/1.1510158.

53 H. Kamada and T. Kutsuwa, Phys.Rev. B 78, 155324 (2008).

54 R. M. Stevenson, R. J. Young, P. Atkinson, K. Cooper, D. A. Ritchie, and A. J. Shields, Nature 439, 179 (2006).

${ }^{55}$ N. Akopian, N. H. Lindner, E. Poem, Y. Berlatzky, J. Avron, D. Gershoni, B. D. Gerardot, and P. M. Petroff, Phys. Rev. Lett. 96, 130501 (2006)
56 C. Santori, D. Fattal, J. Vučković, G. S. Solomon, and Y. Yamamoto, Nature 419, 594 (2002).

57 B. Eble, C. Testelin, P. Desfonds, F. Bernardot, A. Balocchi, T. Amand, A. Miard, A. Lemaître, X. Marie, and M. Chamarro, Phys. Rev. Lett. 102, 146601 (2009)

${ }^{58}$ R. Warburton, Nature Materials 12, 483 (2013).

59 W. B. Gao, A. Imamoglu, H. Bernien, and R. Hanson, Nat. Photonics 9, 363 (2015).

${ }^{60}$ I. Favero and J. M. Gérard, Phys. Rev. B 68, 233301 (2003).

61 A. Reigue, J. Iles-Smith, F. Lux, L. Monniello, M. Bernard, F. Margaillan, A. Lemaitre, A. Martinez, D. P. S. McCutcheon, J. Mørk, R. Hostein, and V. Voliotis, Phys. Rev. Lett. 118, 233602 (2017)

62 T. Grange, N. Somachi, C. Anton, L. D. Santis, G. Coppola, V. Giesz, A. Lemaître, I. Sagnes, A. Auffèves, and P. Senellart, Phys. Rev. Lett 118, 253602 (2017).

${ }^{63}$ C. Roy and S. Hughes, Phys. Rev. Lett. 106, 247403 (2011).

64 J. H. Prechtel, A. V. Kuhlmann, J. Houel, L. Greuter, A. Ludwig, D. Reuter, D. Wieck, and R. J. Warburton, Phys. Rev. X 3, 041006 (2013).

65 R. Malein, T. Santana, J. Zajac, A. Dada, E. Gauger, P. Petroff, J. Lim, J. Song, and B. Gerardot, Phys. Rev. Lett. 116, 257401 (2016).

66 O. Verzelen, R. Ferreira, and G. Bastard, Phys. Rev. B 62, R4809 (2000).

67 S. Ates, S. M. Ulrich, S. Reitzenstein, A. Löffler, A. Forchel, and P. Michler, Phys. Rev. Lett. 103, 167402 (2009).

or A. V. Kuhlmann, J. Houel, D. Brunner, A. Ludwig, D. Reuter, A. D. Wieck, and R. J. Warburton, Rev. Sci. Instrum. 84, 073905 (2013).

${ }^{9}$ A. Högele, S. Seidl, M. Kroner, K. Karrai, R. J. Warburton, B. D. Gerardot, and P. M. Petroff, Phys. Rev. Lett. 93, $217401(2004)$

${ }^{\prime}$ S. Seidl, M. Kroner, P. A. Dalgarno, A. Högele, J. M. Smith, M. Ediger, B. D. Gerardot, J. M. Garcia, P. M. Petroff, K. Karrai, and R. J. Warburton, Phys. Rev. B 72, 195339 (2005)

${ }^{\prime}$ A. N. Vamivakas, M. Atatüre, J. Dreiser, S. T. Yilmaz, A. Badolato, A. K. Swan, B. B. Goldberg, A. Imamoğlu, and M. S. Ünlü, Nano Letters 7 (2007).

72 A. J. Ramsay, A. V. Gopal, E. M. Gauger, A. Nazir, B. W. Lovett, A. M. Fox, and M. S. Skolnick, Phys. Rev. Lett. 104, $017402(2010)$

3 A. J. Ramsay, T. M. Godden, S. J. Boyle, E. M. Gauger, A. Nazir, B. W. Lovett, A. M. Fox, and M. S. Skolnick, Phys. Rev. Lett. 105, 177402 (2010)

74 M. Metcalfe, S. M. Carr, A. Muller, G. S. Solomon, and J. Lawall, Phys. Rev. Lett 105, 037401 (2010).

${ }^{75}$ H. S. Nguyen, G. Sallen, M. Abbarchi, R. Ferreira, C. Voisin, P. Roussignol, G. Cassabois, and C. Diederichs, Phys. Rev. B 87, 115305 (2013).

76 T. Volz, A. Reinhard, M. Winger, A. Badolato, K. J. Hennessy, E. L. Hu, and A. Imamoğlu, Nature Photonics 6 , 605 (2012).

77 M. N. Makhonin, J. E. Dixon, J. Coles, B. Royall, I. J. Luxmoore, E. Clarke, M. Hugues, M. S. Skolnick, and A. M. Fox, Nano Letters 14, 6997 (2014).

78 G. Reithmaier, M. Kaniber, F. Flassig, S. Lichtmannecker, K. Müller, A. Andrejew, J. Vučković, R. Gross, and J. J. Finley, Nano Letters 15, 5208 (2015).

79 A. Kurzmann, B. Merkel, P. A. Labud, A. Ludwig, A. D. 
Wieck, A. Lorke, and M. Geller, Phys. Rev. Lett. 117, 017401 (2016).

80 E. S. Moskalenko, V. T. Donchev, F. K. Karlsonn, P. O. Holtz, B. Monemar, W. V. Schoenfeld, J. M. Garcia, and P. M. Petroff, Phys. Rev. B 68, 155317 (2003).

${ }^{81}$ R. G. Neuhauser, K. T. Shimizu, W. K. Woo, S. A. Empedocles, and M. G. Bawendi, Phys. Rev. Lett. 85, 3301 (2000).

82 C. Arnold, V. Loo, A. Lemaitre, I. Sagnes, O. Krebs, P. Voisin, P. Senellart, and L. Lanco, Phys. Rev. X 4, 021004 (2014).

83 A. Kurzmann, A. Ludwig, A. D. Wieck, A. Lorke, and M. Geller, Appl. Phys. Lett. 108, 263108 (2016).

84 D. Chen, G. R. Landerand, K. S. Krowpman, G. S. Solomon, and E. B. Flagg, Phys. Rev. B 93, 115307 (2016).

85 A. J. Bennett, R. B. Patel, J. Skiba-Szymanska, C. A. Nicoll, I. Farrer, D. A. Ritchie, and A. J. Shields, Applied Physics Letters , 031104 (2010)

${ }^{80}$ A. Kurzmann, A. Ludwig, A. D. Wieck, A. Lorke, and M. Geller, Nano Lett. 16, 3367 (2016).

87 A. Vamivakas, Y. Zhao, S. Fält, A. Badolato, J. M. Taylor, and M. Atatüre, Phys. Rev. Lett. 107, 166802 (2011).

88 M. Reindl, K. D. Jons, D. Huber, C. Schimpf, Y. Huo, V. Zwiller, A. Rastelli, and R. Trotta, Nano letters 17, 4090 (2017).

89 E. S. Moskalenko, F. K. Karlsonn, V. T. Donchev, P. O. Holtz, B. Monemar, W. V. Schoenfeld, and P. M. Petroff, Nano Lett. 5, 2117 (2005).

90 O. Gazzano, T. Huber, V. Loo, S. Polyakov, E. B. Flagg, and G. S. Salomon, Optica 5, 354 (2018).

91 J. M. Smith, P. A. Dalgarno, R. J. Warburton, A. O. Govorov, K. Karrai, B. D. Gerardot, and P. M. Petroff, Phys. Rev. Lett. 94, 197402 (2005).

92 B. D. Gerardot, D. Brunner, P. A. Dalgarno, P. Öhberg, S. Seidl, M. Kroner, K. Karrai, N. G. Stoltz, P. M. Petroff, and R. J. Warburton, Nature 451 (2008).

93 V. Jovanov, S. Kapfinger, M. Bichler, G. Abstreiter, and J. J. Finley, Phys. Rev. B 84, 235321 (2011).

94 A. J. Bennett, M. A. Pooley, R. M. Stevenson, M. B. Ward, R. B. Patel, A. Boyer de la Giroday, N. Sköld, I. Farrer, C. A. Nicoll, D. A. Ritchie, and A. J. Shields, Nature Physics 6, 947 (2010).

95 A. Reigue, A. Lemaître, C. G. Carbonell, C. Ulysse, K. Merghem, S. Guilet, R. Hostein, and V. Voliotis, Appl. Phys. Lett. 112, 073103 (2018).

96 R. Al-Khuzheyri, A. C. Dada, J. Huwer, T. S. Santana, J. Skiba-Szymanska, M. Felle, M. B. Ward, R. M. Stevenson, I. Farrer, M. G. Tanner, R. H. Hadfield, D. A. Ritchie, A. J. Shields, and B. D. Gerardot, Applied Physics Letters 109, 163104 (2016).

97 N. Somaschi, V. Giesz, L. De Santis, J. C. Loredo, M. P. Almeida, G. Hornecker, S. L. Portalupi, T. Grange, C. Antón, J. Demory, C. Gómez, I. Sagnes, N. D. Lanzillotti-Kimura, A. Lemaítre, A. Auffeves, A. G. White, L. Lanco, and P. Senellart, Nature Photonics 10, 340 (2016).

98 J. Bylander, I. Robert-Philip, and I. Abram, Eur. Phys.
J. D 22, 295 (2003).

99 P. A. Dalgarno, M. Ediger, B. D. Gerardot, J. M. Smith, S. Seidl, M. Kroner, K. Karrai, P. M. Petroff, A. O. Govorov, and R. J. Warburton, Phys. Rev. Lett. 100, 176801 (2008).

ơ J. Dreiser, M. Atatüre, C. Galland, T. Müller, A. Badolato, and A. Imamoglu, Phys. Rev. B 77, 075317 (2008)

I0I A. V. Kuhlmann, J. H. Prechtel, J. Houel, A. Ludwig, D. Reuter, A. D. Wieck, and R. J. Warburton, Nature Communications 9, 8204 (2015).

102 N. A. J. M. Kleemans, J. van Bree, A. O. Govorov, J. G. Keizer, G. J. Hamhuis, R. Nötzel, A. Y. Silov, and P. M. Koenraad, Nature Physics 6, 534 (2010).

103 C. K. Hong, Z. Y. Ou, and L. Mandel, Phys. Rev. Lett. 59, 2044 (1987)

${ }^{104}$ X. Ding, Y. He, Z.-C. Duan, N. Gregersen, M.-C. Chen, S. Unsleber, S. Maier, C. Schneider, M. Kamp, S. Höfling, C.-Y. Lu, and J.-W. Pan, Phys. Rev. Lett. 116, 020401 (2016).

${ }^{105}$ H. Wang, Z.-C. Duan, Y.-H. Li, S. Chen, J.-P. Li, Y.-M. He, M.-C. Chen, Y. He, X. Ding, C.-Z. Peng, C. Schneider, M. Kamp, S. Höfling, C.-Y. Lu, and J.-W. Pan, Phys. Rev. Lett. 116, 213601 (2016)

106 M. Delbecq, T. Nakajima, P. Stano, T. Otsuka, S. Amaha, J. Yoneda, K. Takeda, G. Allison, A. Ludwig, A. Wieck, et al., Phys. Rev. Lett. 116, 046802 (2016).

107 E. M. Purcell, Phys. Rev. 69 (1946)

108 F. Liu, A. J. Brash, J. O'Hara, L. M. P. P. Martins, C. L. Phillips, R. J. Coles, B. Royall, E. Clarke, C. Bentham, N. Prtljaga, et al., Nature nanotechnology 13, 835 (2018).

109 J. Liu, K. Konthasinghe, M. Davanço, J. Lawall, V. Anant, V. Verma, R. Mirin, S. W. Nam, J. D. Song, B. Ma, Z. S. Chen, H. Q. Ni, Z. C. Niu, and K. Srinivasan, Phys. Rev. Applied 9, 064019 (2018)

110 In the case of QDs embedded in cavities with high quality factors, cavity quantum electrodynamics effects take place, light-matter interaction is enhanced and the radiative recombination can be accelerated by the Purcell factor.

111 We will avoid using the common terminology of exciton for a photocreated e-h pair, mainly because the confinement potential is dominant compared to Coulomb interaction in strongly confined QDs. This has important consequences on many aspects, like, on the binding mechanism, the interaction with additional carriers, the coupling to photon and the photo-absorption mechanism. We will then use the terminology of "e-h pair", or trio for a charged pair.

112 In the case of spectral diffusion, the total intensity is the convolution of the spectral distribution-assumed to be an homogeneously broadened lorentzian-and the energy shifts distribution of the emission line-assumed to be a gaussian. The FT spectrum is then the product of a gaussian function, characterized by the distribution width and an exponential decaying function, characterized by the coherence time $T_{2}=2 T_{1}$. This is called a Voigt function. 